\title{
Uso de localizadores para personas mayores
}

\author{
Glòria Ribas Miquel \\ Diplomada en enfermería, ABS Cassà de la Selva, IAs, España \\ gloria.ribas@ias.scs.es \\ Alba Pérez-González \\ Psicóloga, Barcelona, España \\ Marta Beltran Vilella \\ Médico de familia, ABS Cassà de la Selva, IAs, España \\ Esther Boix Roqueta \\ Diplomada en enfermería, ABS Cassà de la Selva, IAs, España \\ Montserrat Ferré Munté \\ Diplomada en enfermería, ABS Cassà de la Selva, IAs, España \\ Glòria Reig Garcia \\ Diplomada en enfermería, ABS Cassà de la Selva, IAs, España
}

Josep M. ${ }^{a}$ Gifre Hipòlit

Médico de familia, ABS Cassà de la Selva, IAs, España

Arantza del Valle Gómez

Doctora en psicología, Universitat de Girona, España

\begin{abstract}
Descriptive cross-sectional study which uses self-administered surveys to evaluate the data from three independent samples: professionals, relatives of users of the tracking device and relatives of users who do no use it. Methods: specific survey for 30 professionals. For 7 relatives of users who make use of the device: Quebec survey (QUEST 2.0), Zarit scale and caregiver strain index. For 7 relatives of users who do not make use of the device: Zarit Scale and caregiver strain index. Results: $20 \%$ of the professionals are aware of the existence of some kind of GPs device, only $13.3 \%$ can specify the sort of GPS. The benefits with the highest score in the Liker scale from 0 to 5 are the tranquillity of the family (4.60), followed by the need of information (4.46), and the item with the lowest score is the tranquillity of the user (3.79). Among those patients carrying a tracking device, $85.71 \%$ are men, whereas this percentage turns to a $71,43 \%$ among those who do not carry a tracking device. The burden degree of caregivers is an average of $77.86 \%$ in those who do not use a tracking device, whereas it goes down to $66.58 \%$ in those who do. Caregivers using the tracking device get a $57.1 \%$ of greater burden, and a $14.3 \%$ of minimum burden. Caregivers who do not use the tracking device get a $100 \%$ of greater burden. In the strain index test the results show that a $92.9 \%$ of caregivers reach high levels of burden, whereas for $7.1 \%$ there was no strain at all. $84.6 \%$ of caregivers are women. Conclusions: professionals do not know the tracking device, and the burden of caregivers decreases when they use it. The use of the tracking device should be discussed in an ethical debate by professionals of all areas and relatives of patients.
\end{abstract}

Keyworkds: GPS personal locator, Alzheimer, topographic orientation 


\section{Resumen}

Estudio descriptivo transversal que evalúa, mediante encuestas autoadministrada, los datos procedentes de tres muestras independientes: profesionales, familiares de usuarios que utilizan el dispositivo de localización y familiares de usuarios que no lo utilizan. Métodos: encuesta especifica para 30 profesionales. Para 7 familiares de usuarios que utilizan el dispositivo: encuesta Quebec (QUEST 2.0), escala Zarit y el índice de esfuerzo del cuidador. Para 7 familiares que no utilizan el dispositivo: la escala Zarit y el Índice de esfuerzo del cuidador. Resultados: el $20 \%$ de los profesionales admite conocer algún dispositivo GPS, tan solo un 13,3\% saben especificar el tipo de GPS. Los beneficios mejor puntuados en la escala Liker 0 a 5 son tranquilidad de la familia $(4,60)$, seguido de la necesidad de información $(4,46)$, y el ítem menos valorado la tranquilidad del usuario $(3,79)$. Los pacientes que llevan localizador, los hombres representan un $85,71 \%$ y en los que no llevan localizador representan un 71,43\%. El grado de sobrecarga que tienen los cuidadores obtiene una media de 77,86 \% para los que no utilizan el localizador, frente a un 66,58 \% para los que sí utilizan el localizador. Los cuidadores que utilizan el dispositivo obtienen un 57,1 \% de sobrecarga intensa, un 14,3\% sobrecarga leve. Los cuidadores que no utilizan el localizador obtienen un 100 \% de sobrecarga intensa. En los resultados del test de índice de esfuerzo, el 92,9\% de los cuidadores tienen puntuaciones elevadas de esfuerzo, frente al 7,1 $\%$ que no presentan esfuerzo. De los cuidadores, el 84,6 \% son mujeres. Conclusiones: los profesionales no conocen el dispositivo y la sobrecarga en los cuidadores disminuye con el uso del localizador. Debería plantearse un debate ético entre los diferentes colectivos de profesionales y familiares sobre su uso.

Palabras clave: localizador personal GPS, Alzheimer, orientación topográfica

\section{Introducción}

La desorientación topográfica se inicia de manera mucho más precoz que los primeros indicios de la enfermedad de Alzheimer. Es un deterioro selectivo de las habilidades espaciales, siendo una alteración encontrar el camino en una ruta conocida.

La orientación geográfica y topográfica es la capacidad de establecer la dirección y la distancia entre lugares distantes (orientación alocéntrica) y depende de otras funciones como la atención visual, la memoria de trabajo espacial y la percepción visual-espacial; es por eso que muchas áreas del cerebro están relacionadas con ella. La orientación egocéntrica es, respecto a uno, mismo dónde está el sujeto situado respeto al objeto y como cambia si él se mueve (Grossi, Fasanaro, y otros, 2007).

Las familias de las personas con demencia toman decisiones coherentes con la historia personal y familiar del anciano y también ponen acento en el principio de interés superior de la persona anciana sobre la autonomía. Los cuidadores encuentran la experiencia de toma de decisiones sobre su familiar muy difícil. Consideran que la decisión de utilizar el dispositivo se encuentra dentro de la familia y los profesionales tan solo podrán orientar. La aceptabilidad del dispositivo dependerá del peso, la medida y la facilidad de uso (Ladau, Auslander, y otros, 2011).

Las discapacidades intelectuales y las enfermedades cognitivas asociadas a la demencia constituyen una gran amenaza para el bienestar de los adultos ancianos (Shoval, Auslander, y otros, 2008).

La enfermedad de Alzheimer consta de tres fases: inicial, media y final, siendo parcialmente dependientes en la fase inicial y al inicio de la media, pasando a ser totalmente dependientes para las actividades de la vida diaria (AVD) al final de la segunda fase y la final (De la Vega y Zambrano, 2012).

La enfermedad de Alzheimer no es curable y está estrechamente ligada al envejecimiento. La mayoría de pacientes con Alzheimer viven en casa con la ayuda permanente de un cuidador familiar, mayoritariamente el cónyuge y los hijos. La enfermedad cursa con la pérdida de memoria y una lenta desintegración de la personalidad y el control físico, con manifestaciones como: agresividad, vagar, incontinencia, desinhibición, comer en exceso, alucinaciones, delirios y depresión, entre otras. A medida que la enfermedad se desarrolla, los pacientes se vuelven totalmente dependientes de sus cuidado- 
res, que pueden desarrollar agotamiento físico más o menos grave, pensamientos negativos y estado depresivo y se ven amenazados por un riesgo más alto de mortalidad (Rialle, Ollivet, y otros, 2008).

Los pacientes tienen muchas necesidades durante la progresión de la enfermedad, desde el soporte a la memoria en la demencia leve, a un soporte casi en todos los aspectos funcionales diarios en la demencia severa. Los cuidadores, amigos y vecinos cubren algunas de estas necesidades; sin embargo, $\mathrm{y}$ a pesar de los esfuerzos, no todas las necesidades de una persona con demencia pueden ser cubiertas, ya sea por la falta de tiempo de los cuidadores o por la falta o escasa disponibilidad de servicios profesionales adaptados específicamente a las necesidades concretas de las personas con demencia. Como señalan Laurikis, Reinersmann y otros, (2007), la mayoría de la gente no sabe dónde ir a pedir ayuda, ni que han de tomar la iniciativa para encontrarla, les falta tiempo yendo detrás de su familiar.

El comportamiento errante en las personas con demencia se estima entre un 20-25\%, este problema afecta a la familia y es un comportamiento perturbador relacionado con una mayor carga en las curas y la depresión, es un comportamiento difícil de manejar y es el factor clave para institucionalizar al paciente (Auslander, Werner, y otros, 2010).

Las personas que se pierden tienen un $50 \%$ de posibilidades de sobrevivir si son localizadas durante las primeras 24 horas. A medida que el numero de pacientes con Alzheimer aumente, también lo hará la conducta errante, hecho que hará que la demanda de servicios de búsqueda y rescate y los gastos que están relacionados también aumenten exponencialmente (Edward, 2010).

Algunas de las primeras manifestaciones de los cambios en los hábitos de movilidad asociadas a la demencia, especialmente en la enfermedad de Alzheimer, incluyen problemas en la orientación y los patrones de movilidad, incluyendo el comportamiento errante. Los problemas de movilidad al aire libre son relativamente comunes en personas con demencia y muy a menudo consideradas como una carga (Oswald, Wahl, y otros, 2010).
Los datos indican que más del $70 \%$ de las personas con deterioro cognitivo se desvían de su residencia en algún momento durante el transcurso de la enfermedad (Edward, 2010).

Las nuevas tecnologías de rastreo mediante GPS (sistema global de posicionamiento) pueden ayudar a las familias a tener mayor tranquilidad, a saber que no podrán perder a su familiar. Es un sistema menos restrictivo que a la vez les proporciona más autonomía a las personas con deterioro cognitivo o Alzheimer, al mismo tiempo que da más libertad a los cuidadores para poder realizar otras tareas (Blaschke, Freddolino y otros, 2009).

\section{Proposito del estudio}

Estudio descriptivo transversal que evalúa, mediante encuestas autoadministradas, los datos procedentes de tres muestras independientes: profesionales, familiares de usuarios que utilizan el dispositivo de localización y familiares de usuarios que no lo utilizan.

\section{Metodos usados}

Se diseñó una encuesta para los profesionales de áreas básicas de salud (ABS) la cual fue contestada por una $n=30$. El porcentaje de mujeres fue de 86,7 $\%$. La media de edad fue de 41,30 años (DS=8,437). Por profesiones, el grupo estaba compuesto por un $23,3 \%$ de médicos y un 76,7 \% de enfermeras.

Para los 7 familiares de usuarios que utilizan el dispositivo: encuesta Quebec (QUEST 2.0), escala Zarit y el índice de esfuerzo del cuidador. Para los 7 familiares de usuarios que no utilizan el dispositivo la Escala Zarit y el Índice de esfuerzo del cuidador. Todos los pacientes tenían un nivel inicial de Alzheimer.

\section{Resultados}

En la encuesta específica para los profesionales, en referencia al conocimiento de localizadores mediante GPS, el $20 \%$ admite conocer algún dispositivo de GPS, sin embargo, tan solo un 13,3\% sabe especificar el tipo de GPS; de estos profesionales que saben especificar el nombre del GPS, el 16,7 \% 
tiene algún paciente que actualmente utiliza el dispositivo.

Los beneficios que mejor puntúan los profesionales en escala Likert 0 a 5 son: la tranquilidad de la familia, con una media de 4,60 (DS=0,83), seguido de la necesidad de información con 4,46 ( $\mathrm{DS}=0,83$ ), la satisfacción con un 4,29 (DS=1,50) y, en último lugar, el ítem tranquilidad del usuario, con una media de 3,79 (Ds=1,42).

Se ha buscado la correlación entre los estadísticos tranquilidad para la familia y tranquilidad para el usuario dando un resultado positivo de $\mathrm{r}=0,87$, $(\mathrm{p}=0,05)$.

En cuanto a los resultados obtenidos mediante la encuesta Zarit, cabe especificar que esta se aplicó a una muestra de $n=14$ sobre la que se dividieron dos grupos, uno para los usuarios del dispositivo $(n=7)$ y otro para las personas que no lo utilizan $(n=7)$.

De los pacientes que llevan localizador, los hombres representan un $85,71 \%$ y las mujeres 14,29 $\%$; en los pacientes que no llevan localizador, los hombres representan un $71,43 \%$ y las mujeres un $28,57 \%$.

El grado de sobrecarga que tienen los cuidadores según si utilizan el dispositivo o no obtiene una media de 77,86 (DS=7,06) para los que no utilizan, frente a un 66,58 (DS=14,30) para los que sí llevan localizador. El contraste T-Student para la comparación de medias en muestras independientes proporciona una $\mathrm{t}=3,53, \mathrm{p}=0,04$ indicando que existe una diferencia estadísticamente significativa entre las medias de los grupos.

Los cuidadores que utilizan el dispositivo obtienen un $57,1 \%$ de sobrecarga intensa, un $14,3 \%$ de sobrecarga leve y un $28,6 \%$ no tiene sobrecarga. Los cuidadores que no utilizan el localizador obtienen un $100 \%$ de sobrecarga intensa.

Por sexos, las mujeres tienen mayor porcentaje de sobrecarga intensa un $83,3 \%$ frente al $50 \%$ de los hombres.
En los resultados del test de índice de esfuerzo, el 92,9\% de los cuidadores tienen puntuaciones elevadas de esfuerzo, frente al 7,1\% que no presentan esfuerzo. Por sexos, del 92,9\% que presentan esfuerzo el $84,6 \%$ son mujeres y un $15,4 \%$ son hombres.

\section{Conclusiones}

El grado de sobrecarga de los cuidadores que no utilizan los dispositivos de localización GPs es más grande que los que sí utilizan el dispositivo.

Los cuidadores que no utilizan el localizador presentan más problemas de dolores musculares y ansiedad.

Un aspecto que se debe estudiar en profundidad son los beneficios que proporciona el localizador a los familiares y pacientes.

Todos los resultados encontrados concuerdan con los diferentes artículos consultados, no pudiéndose extrapolar a la población en general por la medida de la muestra.

Hay que promocionar y promover su uso, divulgando sus ventajas e inconvenientes entre los profesionales, para que puedan asesorar a sus pacientes $\mathrm{y}$ familiares antes de que puedan necesitar utilizarlo, pudiendo tomar la decisión de utilizarlo cuando aún están en plenas facultades para hacerlo.

Debería plantearse un debate ético entre los diferentes colectivos de profesionales y familiares sobre su uso.

\section{Limitaciones y aproximaciones más importantes}

Las limitaciones de este estudio son propiamente las derivadas de un diseño transversal: solo permite estudiar asociaciones entre las variables sin posibilidad de establecer casualidad.

Encontrar una muestra bastante representativa por la ley de protección de datos. 


\section{Referencias}

Auslander, G. K., Werner, S., Shoval, N., Isaacson, M., Landau, R. y Heinik, J. (2010). Cooperation with Complex Research Protocols: The Use of Global Positioning Systems with Cognitively Impaired Elders - A Preliminary Report. Open Longevity Science, 4, 1-8.

Blaschke, C. M., Freddolino, P. P. Mullen, E. E. (2009). Ageing and Technology: A Review of the Research Literature. British Journal of Social Work, 39, 641-656.

De la Vega, R. y Zambrano, A. (22 de enero del 2012) Escala de deterioro global (GDs) [en línea]. Disponible en: http:// www.hipocampo.org/reisberg.asp

Grossi, D., Fasanaro, A. M., Cecere, R., Salzano, S. y Trojano, L. (2007). Progressive topographical disorientation: a case of focal Alzheimer's disease. Neurological Science, 25, 107-110.

Landau, R., Auslander, G. K., Werner, S., Shoval, N. y Heinik, J. (2011). Who should make the decision of the use of GPS for people with dementia? Aging \& Mental Health, 15,78-84.

Landau, R., Werner, S., Auslander, G. K., Shoval, N. y Heinik, J. (2009). Attitudes of Family and Professional CareGivers towards the Use of GPS for Tracking Patients with Dementia: An Exploratory Study. British Journal of Social Work, 39, 670-692.

Landau, R., Werner, S., Auslander, G. K., Shoval, N. y Heinik, J. (2010). What do cognitively intact older people think about the use of electronic tracking devices for people with dementia? A preliminary analysis. International Psychogeriatrics, 22, 1301-1309.

Lauriks, S., Reinersmann, A., Van der Roest, H. G., Meiland, F. J., Davies, R. J., Moelaert, F., Mulvenna, M. D., Nugent, C. D. y Dröes, R. M. (2007). Review of ICт-based services for identified unmet needs in people with dementia. Ageing Research Reviews, 6, 223-246.

Mahoney, E. L. y Mahoney, D. F. Acceptance of Wearable Technology by People With Alzheimer's Disease: Issues and Accomodations. (2010). American Journal of Alzheimer's Disease \& Other Dementias, 25, 527-531.

Oswald, F., Wahl, H. W., Voss, E., Schilling, O., Freytag, T., Auslander, G., Shoval, N., Heinik, J. y Landau, R. (2010). The Use of Tracking Technologies for the Analysis of Outdoor Mobility in the Face of Dementia: First Steps into a Project and Some Illustrative Findings From Germany. Journal of Housing for the Elderly, 24, 55-73.

Rialle, V., Ollivet, C., Guigui, C. y Hervé, C. (2008). What do family caregivers of Alzheimer's disease patients desire in smart home technologies? Contrasted results of a wide survey. Methods in Information in Medicine, 47, 63-69.

Shoval, N., Auslander, G. K., Freytag, T., Landau, R., Oswald, F., Seidl, U., Whal, H., Werner, S. y Heinik, J. (2008). The use of advanced tracking technologies for the analysis of mobility in Alzheimer's disease and related cognitive diseases. BMC Geriatrics, 8: 7. 